\title{
A OCORRÊNCIA DA TUBERCULOSE EM UM DISTRITO ADMINISTRATIVO DO MUNICÍPIO DE SÃO PAULO
}

\author{
The presence of tuberculosis in an administrative district of são paulo \\ La ocurrencia de tuberculosis en un distrito administrativo de são paulo
}

Paula Hino ${ }^{1}$

Renata Ferreira Takahashi

Maria Rita Bertolozzi ${ }^{3}$

Emiko Yoshikawa Egry ${ }^{4}$

\section{RESUMO}

0 objetivo do estudo foi caracterizar a situação clínica e epidemiológica dos casos de tuberculose no distrito administrativo do Capão Redondo, Município de São Paulo, para o período de 2000 a 2009. Tratou-se de uma série histórica que utilizou dados secundários do Tbweb. Foram realizadas análises descritivas das variáveis: ano da notificação, sexo, faixa etária, escolaridade, tipo de caso, forma clínica, sorologia para o HIV, tipo de supervisão e desfecho do caso. Observou-se predomínio no sexo masculino, adultos jovens, caso novo e forma pulmonar, sendo que $10 \%$ dos casos estavam infectados pelo HIV. A interpretação dos resultados apresentados evidenciou que as metas propostas para o controle da tuberculose ainda não foram alcançadas no que se refere à cura e ao abandono do tratamento. No entanto, observaram-se esforços dos serviços de saúde no controle da doença por meio do aumento da cobertura do tratamento supervisionado.

Palavras-chave: Tuberculose. Epidemiologia. Saúde pública

\begin{abstract}
The aim of this study was to characterize the clinical and epidemiological patterns of tuberculosis in the district of Capão Redondo, Municipality of Sao Paulo during the period of 2000 up to 2009. It was a historical series that used secondary data from Tbweb. Descriptive analysis of the variables were realized: year of notification, sex, age, education, type of case, clinical, serological testing for HIV, type of supervision and outcome of the case. There was a predominance of males, young adults, new case pulmonary tuberculosis, being $10 \%$ of cases infected with hiv. The interpretation of the results presented showed that the proposed targets for tuberculosis control have not been achieved regarding to healing and treatment dropout. However, it was observed the efforts from health services in disease control through the increased of the coverage on supervised treatment.
\end{abstract}

Keywords: Tuberculosis. Epidemiology. Public health
Resumen

El propósito de este estudio fue caracterizar la situación clínica y epidemiológica de casos de tuberculosis en el distrito administrativo de Capão Redondo, distrito de São Paulo, en el período de 2000 a 2009. Fue una serie que utiliza datos secundarios de Tbweb. Se realizó un análisis descriptivo de las variables: año de la notificación, sexo, edad, escolaridad, tipo de caso, la presentación clínica, el estado del VIH, el tipo de supervisión y resolución del caso. Hubo un predominio de varones, adultos jóvenes, un nuevo caso pulmonar, el 10\% de los casos se infectaron con el VIH. La interpretación de los resultados presentados muestra que las metas propuestas para el control de la tuberculosis no se han logrado con respecto a la cura y el abandono del tratamiento. Sin embargo, hay esfuerzos de los servicios de salud en el control de la enfermedad a través de una mayor cobertura de tratamiento supervisado.

Palabras clave: Tuberculosis. Epidemiologia. Salud publica

${ }^{1}$ Enfermeira. Pós-Doutora pelo Departamento de Enfermagem em Saúde Coletiva da Escola de Enfermagem da USP. São Paulo-SP. Brasil. Emailpaulahino@yahoo.com.br; ²Enfermeira. Profa. Associada do Departamento de Enfermagem em Saúde Coletiva da Escola de Enfermagem da USP. São Paulo-SP. Brasil. Email- rftakaha@usp.br; ${ }^{3}$ Enfermeira Profa. Associada do Departamento de Enfermagem em Saúde Coletiva da Escola de Enfermagem da USP. São Paulo-SP. Brasil. Email- mrbertol@usp.br; ${ }^{4}$ Enfermeira. Profa. Titular do Departamento de Enfermagem em Saúde Coletiva da Escola de Enfermagem da USP. São Paulo-SP. Brasil. Email- emiyegry@usp.br. 


\section{INTRODUÇÃO}

Embora a tuberculose (Tb) seja uma doença prevenível, curável na quase totalidade dos casos, e o tratamento seja custeado pelo sistema público, observa-se sua recrudescência e consequente repercussão nos níveis de saúde e mortalidade, mantendo sua relevância nacional e internacional como problema de saúde pública ${ }^{1}$.

Vários aspectos devem ser considerados para a manutenção da Tb em nosso meio, destacando-se o fator social como determinante principal associado à ocorrência da doença. As condições de pobreza, que resultam em dificuldades de acesso aos serviços de saúde, envelhecimento da população, urbanização desordenada e processos migratórios, além do surgimento da epidemia de HIV/AIDS na década de 1980 são apontados como obstáculos no controle da $\mathrm{Tb}^{2}$. Além disso, tanto a ingestão irregular dos medicamentos quanto a não adesão ao tratamento favorecem o desenvolvimento da multidrogarresistênsia, o que compromete ainda mais o sucesso do tratamento ${ }^{1}$.

0 Global Tuberculosis Control é um relatório publicado anualmente pela Organização Mundial da Saúde (OMS) que traz informações recentes sobre a Tb e os progressos realizados nos níveis global, regional e nacional. O Plano Global para o Combate da Tuberculose 2011-2015 tem como objetivo principal reduzir drasticamente a carga da doença até 2015; para isso, foram propostos os seguintes componentes: expandir a estratégia Directly Observed Therapy (DOTS), visar a coinfecção Tb/HIV, Tb multirresistente e as necessidades de saúde de populações pobres e vulneráveis, fortalecer a atenção primária à saúde, empoderar as pessoas com Tb e a sociedade organizada, envolver todos os prestadores de serviços de saúde e incentivar as pesquisas ${ }^{3}$. 0 relatório de 2010 informa que a Parceria Stop TB fixou duas metas adicionais: reduzir pela metade as taxas de prevalência e mortalidade até $2015 \mathrm{em}$ comparação aos níveis de 1990 e eliminar a Tb como problema de saúde pública até $2050^{4}$.

0 Brasil é um dos 22 países responsáveis por $80 \%$ da carga mundial da Tb. Segundo dados da OMS para o ano de 2009 , foram diagnosticados $86 \%$ dos casos estimados, sendo notificados 75.040 casos, destes 39.267 bacilíferos. Em relação ao resultado de tratamento, $71 \%$ dos doentes foram curados, 8,3\% abandonaram o tratamento, 4,5\% foram a óbito ${ }^{5}$. Observa-se que a distribuição geográfica da Tb no País concentra-se nos grandes centros urbanos, como capitais dos estados e regiões metropolitanas. A região Sudeste do Brasil representa o segundo lugar em taxa de incidência da Tb no Brasil, com 37,6 casos/100.000 habitantes), perdendo apenas para a região Norte $(45,2 / 100.000 \text { habitantes) })^{3}$. Segundo dados da Divisão de Controle da Tb, do Centro de Vigilância Epidemiológica, o Estado de São Paulo notificou, no ano de $2010,16.165$ casos novos, sendo que $83,4 \%$ apresentaram a forma pulmonar da doença. No mesmo ano, o Município de
São Paulo registrou um total de 6.830 casos, sendo 5.858 casos novos, 394 recidivas, 502 retratamentos após abandono, 66 retratamentos por falha do tratamento.

Os dados revelam um grande desafio para o Brasil em relação às metas pactuadas desde 1999 pelo Ministério da Saúde (MS), de diagnosticar pelo menos $90 \%$ dos casos esperados, alcançar $85 \%$ de cura dos casos diagnosticados e reduzir o abandono para 5\%. Conforme verificado, o País está abaixo dessas metas e requer melhoria do acesso ao diagnóstico e tratamento da $\mathrm{Tb}$, bem como as taxas de adesão ao tratamento, entre outras ações ${ }^{4}$.

Em 2006, o Pacto pela Saúde integrou três eixos: pela Vida, em Defesa do SUS e o de Gestão. 0 Pacto pela Vida prevê o fortalecimento da capacidade de respostas às doenças emergentes e endemias, com ênfase na dengue, hanseníase, malária, influenza e Tb. As metas para o controle da Tb apontam para a necessidade de se atingir pelo menos $85 \%$ de cura dos casos novos de baciliferos diagnosticados a cada ano, conforme meta do MS 6 .

Considerando a magnitude da Tb como um problema de saúde pública, este estudo teve como objetivo descrever as características clínicas e epidemiológicas dos casos de Tb notificados em um período de dez anos (2000 a 2009) em um distrito administrativo do Município de São Paulo.

\section{MÉTODO}

Trata-se de um estudo descritivo, de caráter retrospectivo, que fez uso de dados secundários. A população do estudo foi constituída por pessoas portadoras de Tb, de todas as formas clínicas e residentes no distrito administrativo do Capão Redondo e que foram notificados no período de 01 de janeiro de 2000 a 31 de dezembro de 2009. Foram excluídos os casos que apresentavam duplicidade de notificação, mudança de diagnóstico e aqueles que pertenciam ao sistema penitenciário.

0 cenário do estudo foi Capão Redondo, um distrito administrativo localizado na região Sul do Município de São Paulo. Segundo dados do site da Prefeitura de São Paulo, o Capão Redondo possui uma área de 13,8 km² e contabilizava uma população de 272.103 habitantes no ano de 2009.

As variáveis de interesse do estudo foram: ano da notificação, sexo, faixa etária, escolaridade, tipo de caso, forma clínica, sorologia para o HIV, tipo de supervisão e desfecho do caso.

Os dados referentes aos casos de Tb foram extraídos do TBWeb no mês maio de 2010, e, para o cálculo dos Coeficientes de Incidência, consultou-se a população de cada ano estudado, pelo site da prefeitura do Município de São Paulo. A partir de 2006, 0 TBWeb substituiu um programa que era utilizado no Estado de São Paulo, o EpiTb, possibilitando que as notificações fossem feitas via Internet. OTBWeb, de uso exclusivo do Estado de São Paulo, trabalha com um banco de dados único, o que garante dados mais fidedignos 
e facilita a comunicação entre os diversos níveis de vigilância, pois permite a unificação do registro de cada caso e comunicados automáticos por ocasião de transferências ou hospitalizações ${ }^{7}$.

Foi utilizada uma planilha eletrônica do Excel para armazenamento dos dados coletados, e, posteriormente, foram realizadas técnicas de estatística descritiva para a análise dos dados.

Em se tratando de pesquisa que envolve seres humanos e atendendo aos preceitos ético-legais, a pesquisa atendeu às normas da Resolução n $196 / 96$ e o projeto foi aprovado pelos Comitês de Ética em Pesquisa da Escola de Enfermagem da Universidade de São Paulo (processo 783/2006).

\section{RESULTADOS}

A Tabela 1 apresenta, para o período de 2000 a 2009, o número absoluto e a frequência relativa dos casos novos de Tb, segundo sexo, que foram notificados no Capão Redondo, bem como os respectivos Coeficientes de Incidência. A série histórica da Tb no Capão Redondo contou com 1.612 casos notificados no período de 2000 a 2009; destes, 1.442 foram casos novos de Tb. Em relação à variável sexo, o percentual encontrado foi bem próximo ao observado para os casos novos.

Tabela 1. Percentual de casos novos de tuberculose por sexo e Coeficiente de Incidência de tuberculose. Capão Redondo, 2000 a 2009

\begin{tabular}{|c|c|c|c|c|c|c|}
\hline Ano & & & Sexo & & Total & $\begin{array}{l}\text { Coeficiente de } \\
\text { Incidência }\end{array}$ \\
\hline & $\begin{array}{c}\text { Feminino } \\
\left(n^{*}\right)\end{array}$ & $\%$ & $\begin{array}{c}\text { Masculino } \\
\left(\mathrm{n}^{*}\right)\end{array}$ & $\%$ & & $\begin{array}{c}\text { (casos/100.000 } \\
\text { hab) }\end{array}$ \\
\hline 2000 & 63 & 38,7 & 100 & 61,3 & 163 & 67,8 \\
\hline 2001 & 63 & 36,6 & 109 & 63,4 & 172 & 70,4 \\
\hline 2002 & 70 & 40,9 & 101 & 59,1 & 171 & 68,9 \\
\hline 2003 & 32 & 33,3 & 64 & 66,7 & 96 & 38,1 \\
\hline 2004 & 18 & 33,3 & 36 & 66,7 & 54 & 21,1 \\
\hline 2005 & 45 & 32,1 & 95 & 67,9 & 140 & 51,6 \\
\hline 2006 & 64 & 47,8 & 70 & 52,2 & 134 & 53,3 \\
\hline 2007 & 57 & 35,0 & 106 & 65,0 & 163 & 61,3 \\
\hline 2008 & 71 & 38,4 & 114 & 61,6 & 185 & 68,8 \\
\hline 2009 & 60 & 36,6 & 104 & 63,4 & 164 & 60,3 \\
\hline Total & 543 & 37,7 & 899 & 62,3 & 1442 & 56,2 \\
\hline
\end{tabular}

Observa-se que os casos novos não apresentaram variação significativa em relação ao sexo durante o período estudado, evidenciando predomínio do sexo masculino, que representou $62,3 \%$ do total de casos. A média do Coeficiente de Incidência foi de 56,2 casos/100.000 habitantes, porém mostrou variação de 21,1 casos/100.000 habitantes (em 2004) e 70,4 casos/100.000 habitantes (em 2001).

No que diz respeito à faixa etária, pode-se observar predomínio de casos entre indivíduos de 20 a 49 anos (67\%). É importante destacar um percentual expressivo de casos em indivíduos com 60 anos ou mais anos (8,7\%). Quanto à escolaridade, 100 casos de $\mathrm{Tb}(6,2 \%)$ eram analfabetos, $11 \%$ referiram ter de 1 a 3 anos de estudo, 29,7\% e 26,4\% mencionaram ter de 4 a 7 e 8 a 11 anos de estudo, respectivamente. Apenas 3,7\% do total de casos tinha 12 ou mais anos de estudo. Ressalta-se que, para esta variável em especial, houve um percentual elevado de respostas sem informação $(23,1 \%)$.

A Tabela 2 apresenta o tipo de caso e a forma clínica dos casos de Tb notificados no período de 2000 a 2009 no Capão Redondo.

\begin{tabular}{llll}
\multicolumn{4}{l}{ Tabela 2. Distribuição dos casos de tuberculose segundo tipo de caso e forma clínica. Capão Redondo, 2000 a 2009} \\
\hline \multicolumn{2}{ll}{ Variáveis } & $\mathbf{n}^{\mathbf{0}}$ & $\%$ \\
\hline Tipo de caso & Novo & 1.442 & 89,5 \\
& Recidiva & 90 & 5,6 \\
& Retratamento - abandono & 72 & 4,5 \\
& Retratamento -falha & 8 & 0,5 \\
Forma clínica & & 1275 & 79,1 \\
& Pulmonar & 265 & 16,4 \\
& Extrapulmonar & 72 & 4,5 \\
\hline
\end{tabular}

Fonte: TBWEB, 2010.

Para o período estudado, o tipo de caso predominante foi o caso novo, apresentando 89,5\% do total de casos. Os casos de recidiva representaram um percentual menor, com 90 casos (5,6\%), sendo que $10 \%$ apresentaram sorologia positiva para o HIV. Em seguida, notou-se os casos de retratamento, sendo que em $4,5 \%$, o desfecho foi por abandono do tratamento e $0,5 \%$ por falência do esquema medicamentoso.

Analisando a distribuição percentual de acordo com a forma clínica da Tb, observou-se predomínio da forma pulmonar $(79,1 \%)$, seguida da extrapulmonar (16,4\%). 0 percentual de casos que desenvolveram as duas formas da doença, pulmonar e extrapulmonar, concomitantemente, foi de $4,4 \%$. 
A Tabela 3 ilustrou o tipo de supervisão do tratamento realizado, segundo modalidade de tratamento supervisionado ou autoadministrado. No início do período estudado, a cobertura do tratamento supervisionado foi de apenas $5,1 \%$, sendo observado 0 aumento gradativo no decorrer dos anos, atingindo 86,6\% de cobertura em 2009. No Capão Redondo, até 2004, a maioria dos casos realizava o tratamento autoadministrado.

\begin{tabular}{|c|c|c|c|c|c|c|c|}
\hline Ano & $\begin{array}{l}\text { TS } \\
\left(n^{*}\right)\end{array}$ & $\%$ & $\begin{array}{l}\text { TA } \\
\left(n^{*}\right)\end{array}$ & $\%$ & $\begin{array}{c}\text { Sem inf } \\
\left(n^{*}\right)\end{array}$ & $\%$ & Total \\
\hline 2000 & 9 & 5,1 & 154 & 87,5 & 13 & 7,4 & 176 \\
\hline 2001 & 26 & 13,0 & 165 & 82,5 & 9 & 4,5 & 200 \\
\hline 2002 & 35 & 17,5 & 158 & 79,0 & 7 & 3,5 & 200 \\
\hline 2003 & 28 & 25,9 & 77 & 71,3 & 3 & 2,8 & 108 \\
\hline 2004 & 16 & 27,6 & 37 & 63,8 & 5 & 8,6 & 58 \\
\hline 2005 & 112 & 73,2 & 37 & 24,2 & 4 & 2,6 & 153 \\
\hline 2006 & 111 & 74,5 & 8 & 5,4 & 30 & 20,1 & 149 \\
\hline 2007 & 135 & 76,3 & 30 & 16,9 & 12 & 6,8 & 177 \\
\hline 2008 & 162 & 79,4 & 30 & 14,7 & 12 & 5,9 & 204 \\
\hline 2009 & 162 & 86,6 & 20 & 10,7 & 5 & 2,7 & 187 \\
\hline Total & 796 & 49,4 & 716 & 44,4 & 100 & 6,2 & 1612 \\
\hline
\end{tabular}

Observa-se maior ênfase no tratamento supervisionado a partir de 2005, quando este tipo de supervisão atingiu $73,2 \%$ dos casos.
Em relação à sorologia para o HIV, $162(10 \%)$ casos de Tb estavam infectados pelo HIV (Tabela 4).

Tabela 4. Distribuição dos casos de tuberculose segundo sorologia para HN. Capão Redondo, 2000 a 2009.

\begin{tabular}{|c|c|c|c|c|c|c|c|c|c|}
\hline Ano & $\begin{array}{c}\text { Positivo } \\
\left(\mathrm{n}^{*}\right)\end{array}$ & $\%$ & $\begin{array}{c}\text { Negativo } \\
\left(n^{*}\right)\end{array}$ & $\%$ & $\begin{array}{c}\text { Nāo } \\
\text { realizado } \\
\left(n^{*}\right)\end{array}$ & $\%$ & $\begin{array}{l}\text { Sem } \\
\text { inf } \\
\left(n^{*}\right)\end{array}$ & $\%$ & Total \\
\hline 2000 & 16 & 9,1 & 113 & 64,2 & 39 & 22,2 & 8 & 4,5 & 176 \\
\hline 2001 & 23 & 11,5 & 128 & 64,0 & 45 & 22,5 & 4 & 2,0 & 200 \\
\hline 2002 & 18 & 9,0 & 133 & 66,5 & 48 & 24,0 & 1 & 0,5 & 200 \\
\hline 2003 & 16 & 14,8 & 60 & 55,6 & 29 & 26,9 & 3 & 2,8 & 108 \\
\hline 2004 & 7 & 12,1 & 34 & 58,6 & 17 & 29,3 & 0 & 0,0 & 58 \\
\hline 2005 & 13 & 8,5 & 122 & 79,7 & 14 & 9,2 & 4 & 2,6 & 153 \\
\hline 2006 & 20 & 13,4 & 103 & 69,1 & 21 & 14,1 & 5 & 3,4 & 149 \\
\hline 2007 & 18 & 10,2 & 140 & 79,1 & 18 & 10,2 & 1 & 0,6 & 177 \\
\hline 2008 & 13 & 6,4 & 179 & 87,7 & 11 & 5,4 & 1 & 0,5 & 204 \\
\hline 2009 & 18 & 9,6 & 160 & 85,6 & 7 & 3,7 & 2 & 1,1 & 187 \\
\hline Total & 162 & 10,0 & 1172 & 72,7 & 249 & 15,4 & 29 & 1,8 & 1612 \\
\hline
\end{tabular}

Do total de casos, observou-se que $249(15,4 \%)$ casos não realizaram a sorologia para HIV. Evidenciou-se um percentual elevado de casos que não realizaram o teste nos primeiros anos da série histórica, mas que foi reduzindo gradativamente no decorrer do período. Já em 2009, apenas

\section{$3,7 \%$ dos casos não realizaram o teste anti-HIV.}

A Tabela 5 apresenta os resultados segundo 0 desfecho do tratamento. Quanto ao desfecho favorável, a média de cura dos casos foi de $74,6 \%$, apresentando variação de $69 \%(2004)$ a $79,7 \%$ (2005).

\begin{tabular}{|c|c|c|c|c|c|c|c|c|c|c|c|c|c|c|c|}
\hline Ano & $\begin{array}{l}\text { Cura } \\
\left(n^{*}\right)\end{array}$ & $\%$ & $\begin{array}{l}\text { Abandono } \\
\left(n^{\circ}\right)\end{array}$ & $\%$ & $\begin{array}{l}\text { Falha do } \\
\text { tratamento } \\
\left(n^{\circ}\right)\end{array}$ & $\%$ & $\begin{array}{l}\text { Óbito } \\
\text { por } \\
\mathrm{Tb}\left(\mathrm{n}^{\circ}\right)\end{array}$ & $\%$ & $\begin{array}{l}\text { Óbito } \\
\text { não } \\
\mathrm{Tb}\left(\mathrm{n}^{\circ}\right)\end{array}$ & $\%$ & $\begin{array}{c}\text { Transferência } \\
\left(n^{*}\right)\end{array}$ & $\%$ & $\begin{array}{l}\text { Sem } \\
\text { inf } \\
\left(n^{\circ}\right)\end{array}$ & $\%$ & Total \\
\hline 2000 & 125 & 71,0 & 24 & 13,6 & 0 & 0,0 & 6 & 3,4 & 10 & 5,7 & 5 & 2,8 & 6 & 3,4 & 176 \\
\hline 2001 & 139 & 69,5 & 34 & 17,0 & 0 & 0,0 & 7 & 3,5 & 8 & 4,0 & 8 & 4,0 & 4 & 2,0 & 200 \\
\hline 2002 & 141 & 70,5 & 27 & 13,5 & 0 & 0,0 & 8 & 4,0 & 11 & 5,5 & 10 & 5,0 & 3 & 1,5 & 200 \\
\hline 2003 & 76 & 70,4 & 9 & 8,3 & 0 & 0,0 & 7 & 6,5 & 7 & 6,5 & 6 & 5,6 & 3 & 2,8 & 108 \\
\hline 2004 & 40 & 69,0 & 5 & 8,6 & 0 & 0,0 & 8 & 13,8 & 4 & 6,9 & 1 & 1,7 & 0 & 0,0 & 58 \\
\hline 2005 & 122 & 79,7 & 13 & 8,5 & 0 & 0,0 & 5 & 3,3 & 7 & 4,6 & 4 & 2,6 & 2 & 1,3 & 153 \\
\hline 2006 & 114 & 76,5 & 10 & 6,7 & 1 & 0,7 & 5 & 3,4 & 16 & 10,7 & 0 & 0,0 & 3 & 2,0 & 149 \\
\hline 2007 & 140 & 79,1 & 12 & 6,8 & 2 & 1,1 & 6 & 3,4 & 13 & 7,3 & 2 & 1,1 & 2 & 1,1 & 177 \\
\hline 2008 & 162 & 79,4 & 17 & 8,3 & 3 & 1,5 & 5 & 2,5 & 11 & 5,4 & 4 & 2,0 & 2 & 1,0 & 204 \\
\hline 2009 & 144 & 77,0 & 12 & 6,4 & 3 & 1,6 & 7 & 3,7 & 9 & 4,8 & 1 & 0,5 & 11 & 5,9 & 187 \\
\hline Total & 1203 & 74,6 & 163 & 10,1 & 9 & 0,6 & 64 & 4,0 & 96 & 6,0 & 41 & 2,5 & 36 & 2,2 & 1612 \\
\hline
\end{tabular}

Como desfecho desfavorável, constatou-se falha do tratamento para nove casos $(0,6 \%)$, sendo os casos distribuídos no período de 2006 a 2009. A média de abandono do tratamento foi considerado elevado $(10,1 \%)$ variando de $6,4 \%$
(2009) a 17\% em 2001.0 percentual de óbitos totalizou $10 \%$ dos casos, sendo que os óbitos ocorridos durante 0 tratamento e que tiveram a Tb como causa básica de óbito representaram $4 \%$ do total. 0 percentual de óbitos que não 
estavam relacionados à $\mathrm{Tb}$ foi de $6 \%$.

Observa-se que 36 casos não continham informação sobre o desfecho do tratamento, e, para 2009, encontram-se 11 casos que, provavelmente, estariam em tratamento no momento da coleta de dados.

\section{DISCUSSÃO}

Os resultados do estudo permitiram o conhecimento do perfil clínico e epidemiológico da Tb no Capão Redondo, para um período de 10 anos.

Observou-se predomínio do sexo masculino em todos os anos, conforme evidenciaram diversos estudos que buscaram caracterizar o perfil de pessoas com Tb ${ }^{2,7-9}$. Apesar de não estarem bem esclarecidos os motivos que levam as pessoas do sexo masculino a adoecerem por Tb mais do que as mulheres, este fato pode ser relacionado a fatores econômicos, culturais e sociais, como a maior exposição ao Mycobacterium tuberculosis ${ }^{8}$.

A queda importante da incidência da Tb observada nos anos de 2003 e 2004 pode estar relacionada a mudanças no quadro dos profissionais de saúde com qualificação para a realização das ações de controle da Tb, tais como: busca ativa de sintomáticos respiratórios e diagnóstico precoce. Outra explicação reside na insuficiência ou na precariedade na organização dos serviços de saúde e na operacionalização das ações que subsidiam o diagnóstico, que vão desde a coleta e fluxo de encaminhamento de material, a demora ou ausência do resultado do exame, até a convocação do usuário para conhecimento do diagnóstico, notificação do caso e início do tratamento.

A ocorrência da Tb em pessoas na faixa etária economicamente ativa também corrobora os achados da literatura ${ }^{2,9-10}$. Isso tem implicações sociais importantes, uma vez que muitos doentes são os provedores do sustento da família e se veem impossibilitados de participar do processo de produção social, dada a debilidade física decorrente da doença e dos efeitos colaterais dos tuberculostáticos.

Em relação aos idosos, encontrou-se neste estudo um percentual considerável de casos de Tb neste grupo etário, que merece atenção especial por parte dos serviços de saúde. Esta situação pode ser explicada pelo crescimento da população idosa como conseqüência do aumento da expectativa de vida e pelo fato de a população idosa de hoje ter vivido em épocas de alta prevalência da doença; tendo sido infectados pelo bacilo, podem vir a desenvolver a doença devido às deficiências próprias do envelhecimento. Acrescido a isso, outros fatores contribuem para acometer esta faixa etária com maior intensidade, como dificuldade de acesso aos serviços de saúde, confinamento de idosos em asilos, demora na procura da assistência médica devida à pouca importância dada aos sintomas, considerados próprios de doenças que acometem os idosos. Nesse sentido, é importante orientar os trabalhadores de saúde quanto aos sinais e sintomas da Tb em indivíduos idosos ${ }^{11}$.

0 fato de $23,1 \%$ dos casos não conterem a informação sobre escolaridade pode elevar ainda mais o percentual de casos com baixa ou nenhuma escolaridade.

Quando se trabalha com dados secundários, deve ser considerada a possível limitação do estudo. Portanto, ressaltase a importância da conscientização dos profissionais de saúde sobre a qualidade e fidedignidade no preenchimento da ficha de notificação compulsória, pois isso permitirá a realização de análises epidemiológicas detalhadas e, assim, o embasamento de decisões gerenciais por parte dos Programa de Controle da Tuberculose, em seus níveis nacional, estadual e municipal ${ }^{12}$.

A forma clínica mais comum da Tb encontrada no estudo foi a pulmonar, que, juntamente com a forma laríngea, é a forma transmissível da doença quando a pessoa se encontra sem o tratamento adequado, merecendo, por isso, atenção especial dos profissionais de saúde; foi observada, também, a ocorrência de 90 casos de recidiva, que pode ter sido influenciada pela coinfecção $\mathrm{Tb} / \mathrm{HIV}$ em $10 \%$ dos casos.

Segundo definição do Novo Manual de Normas para o Controle da Tb, publicado em 2011, a recidiva da Tb é a doença em atividade, já tratada e curada anteriormente, independentemente do tempo decorrido do tratamento anterior ${ }^{13}$. Em um estudo que buscou identificar os fatores de risco para a recidiva da $\mathrm{Tb}$, o uso irregular de medicamentos e a infecção pelo HIV mostraram-se relacionados com taxas maiores deste tipo de caso ${ }^{14}$.

0 predomínio do caso novo corrobora os achados da literatura. Entende-se por caso novo o paciente que nunca usou ou usou por menos de 30 dias medicamentos antituberculose ${ }^{13}$. Ainda segundo o Manual de Recomendações para o Controle da Tuberculose no Brasil, considera-se retorno após abandono quando o doente retorna o tratamento de TB após tê-lo iniciado e deixou de comparecer à unidade de saúde por mais de 30 dias consecutivos a partir da data marcada para seu retorno ou da última tomada supervisionada. A falha do tratamento é a persistência de baciloscopia for temente positiva $(++$ ou +++$)$ desde o início até o quarto mês de tratamento, ou positividade inicial seguida de negativação e nova positividade a partir do quarto mês de tratamento.

A cober tura do tratamento supervisionado vem sendo aumentada gradativamente no Capão Redondo, como pode ser observado nos resultados do estudo. Este tipo de tratamento é um elemento-chave da estratégia Directly Observed Short Course (DOTS), que busca o fortalecimento da adesão ao tratamento e a prevenção de resistência aos medicamentos, reduzindo os casos de abandono e aumentando a probabilidade de cura ${ }^{13}$. Este tipo de supervisão destaca-se como uma potencialidade no controle da Tb, por meio da contribuição nos indicadores epidemiológicos de cura, redução das taxas de abandono do tratamento e da mortalidade, e redução do desenvolvimento de resistência aos medicamentos. 
Além disso, o tratamento supervisionado propicia o estabelecimento de vínculo, do diálogo entre profissionais de saúde e doente; permite identificar necessidades dos doentes; e é tido como espaço para informação sobre a doença e o tratamento ${ }^{15}$.

Apesar de o teste anti-HIV ser uma recomendação do Ministério da Saúde, dada a relação entre a Tb e a infecção pelo HIV, observou-se que $15,4 \%$ dos casos não o realizaram. No entanto, o estudo evidenciou a melhora dos percentuais de realização do exame, considerando que, em 2009, o percentual de casos que não realizaram a sorologia foi de $3,7 \%$.

Devido à elevada ocorrência de coinfecção Tb/HIV no cenário do estudo, e ao fato de a Tb ser considerada a primeira causa de morte em pacientes com AIDS no Brasil, e doentes com coinfecção Tb/HIV terem maior probabilidade de apresentar desfecho desfavorável ao tratamento da Tb, ressaltase que os programas de controle devem trabalhar articulados, ressaltando a importância de capacitação dos profissionais de saúde, que devem estar atentos para os sinais e sintomas da Tb, bem como a importância da realização de sorologia para o HIV ${ }^{10}$.

Face à vigência de quase duas décadas do acesso universal ao tratamento antirretroviral e à elevada prevalência da coinfecção Tb/HIV no Brasil, urge a ampliação da prática de testagem sorológica ao HIV, visando realizar precocemente o diagnóstico e potencializar o impacto na transmissão do HIV, morbidade e mortalidade por AIDS3.

Observou-se que do total de casos que apresentaram sorologia positiva para o HIV, os percentuais de cura, abandono e óbito foram menos favoráveis quando comparados ao grupo que não estava infectado pelo HIV. Constatou-se que a média de cura foi de $47,5 \%$, sendo que o valor mínimo foi de $33,3 \%$ e o máximo foi $56,5 \%$. 0 abandono do tratamento foi de $13 \%$, e os casos que evoluíram para óbito representaram 32,1\% do total de coinfectados.

Os resultados evidenciaram que as taxas de cura $(74,6 \%)$ e abandono $(10,1 \%)$, apesar de terem apresentado melhora nos indicadores no decorrer do período considerado, ainda encontram-se abaixo das metas propostas pelo Ministério da Saúde, que é de curar pelo menos $85 \%$ dos casos e reduzir 0 abandono para $5 \%$.

No entanto, observam-se esforços dos serviços de saúde no controle da enfermidade por meio do aumento da cobertura do tratamento supervisionado, o que reflete impacto positivo nos percentuais de cura e reduz 0 abandono do tratamento.

A Tb é melhor compreendida à luz da Teoria da Determinação Social do Processo Saúde-Doença, pois causa impacto na economia das sociedades e sofrimento nas pessoas e famílias que vivenciam a doença, por apresentar estreita relação com os processos de desigualdades sociais, acometendo grupos de maior vulnerabilidade e segmentos populacionais na faixa etária economicamente ativa ${ }^{16}$.
Um estudo desenvolvido com o objetivo de analisar o desempenho da atenção básica por meio do acesso ao tratamento para Tb em serviços de saúde vinculados à Estratégia Saúde da Família e em ambulatórios de referência identificou que, embora o tratamento da Tb seja ofertado pelo serviço público de saúde, ainda representa um custo econômico para o indivíduo, dada a necessidade de deslocamento até o serviço de saúde, bem como a perda do turno de trabalho para ser consultado. Portanto, éfundamental a investigação dos aspectos organizacionais necessários à efetiva incorporação e sustentabilidade de ações de tratamento da doença na atenção básica, tais como a qualificação de profissionais de saúde para atuarem no controle da $\mathrm{Tb}^{17}$.

Diversos trabalhos apontam fatores que influenciam no desempenho dos serviços de saúde no que se refere às ações de controle da Tb, dentre as quais se destacam: contexto socioeconômico e cultural do usuário, qualidade insuficiente de recursos humanos, rotatividade dos profissionais de saúde, não organização das ações para a deteç̧ão precoce ${ }^{18-19}$.

$\mathrm{A} T \mathrm{~Tb}$ é uma doença que está muito ligada às baixas condições de vida da população, e não há perspectiva a curto prazo de melhoria das desigualdades sociais. A política de combate à enfermidade depende do diagnóstico precoce, do tratamento mais rápido possível e da investigação dos comunicantes como estratégias para controlar a endemia ${ }^{20}$.

\section{CONSIDERAÇÕES FINAIS}

0 estudo permitiu conhecer algumas características clínicas e epidemiológicas dos casos de Tb de um distrito administrativo do Município de São Paulo, notificados no período de 2000 a 2009. Mesmo sendo essa uma região periférica de São Paulo, apresenta semelhanças ao perfil epidemiológico da Tb no País. No entanto, ressalta-se a necessidade de compreender o contexto da ocorrência da $\mathrm{Tb}$, uma vez que a forma como os indivíduos estão alocados na sociedade qualifica, em parte, o acesso aos serviços de saúde e a satisfação de suas necessidades de saúde. Além disso, é indiscutível que a organização dos serviços, no que tange às intervenções voltadas para o controle da Tb, contribui sobremaneira para a identificação e tratamento dos casos.

\section{REFERÊNCIAS}

1- Paixão LMM, Gontijo ED. Perfil de casos de tuberculose notificados e fatores associados ao abandono. Rev. saúde pública. 2007 abr; 41(2): 205-13.

2- Xavier MIM, Barreto ML. Tuberculose na cidade de Salvador, Bahia, Brasil: o perfil na década de 1990. Cad. saúde pública. 2007 fev; 23(2): 445-53. 
3- Ministério da Saúde (BR). Secretaria de Vigilância em Saúde. Boletim Epidemiológico (Especial Tuberculose). 2012; 43 Esp: 1-12.

4- World Health Organization. Global Tuberculosis control. Geneva(SUI): WHO Report; 2010.

5- Hiijaar MA, Gerhardt G, Teixeira GM, Procópio MJ. Retrospecto do controle da tuberculose no Brasil. Rev. saúde pública. 2007; 41Supl. 1:50-8.

6- Ministério da Saúde (BR). Secretaria Executiva. Departamento de Apoio à Descentralização. Coordenação-Geral de Apoio à Gestão Descentralizada. Diretrizes operacionais dos Pactos pela Vida, em Defesa do SUS e de Gestão. Brasilia(DF): Ministério da Saúde; 2006. (SérieA. Normas e Manuais Técnicos)

7- Galesi VMN. Dados de tuberculose do Estado de São Paulo. Rev. saúde pública. 2007; 41Supl. 1:121.

8- Belo MTCT, et al. Tuberculose e gênero em um município prioritário do estado do Rio de Janeiro. J. bras. pneumol. 2010 set/out; 36(5): 621-5.

9- Coelho AGV, Zamarioli LA, Perandones CA, Cuntiere I, Waldman EA. Características da tuberculose pulmonary emárea hiperendêmica- município de Santos (SP). J. bras. pneumol. 2009 out; 35(10): 998-1007.

10- Hino P, Cunha TN, Villa TCS, Santos CB. Perfil dos casos novos de tuberculose notificados em Ribeirão Preto (São Paulo) no período de 2000 a 2006. Ciênc. saúde coletiva. 2011; 16 Supl 1: 1295-301.

11- Cavalcanti ZR, Albuquerque MFPM, Campelo ARL, Ximenes RAA, Montarroyos U, Verçosa MKA. Característica da Tuberculose em idosos no Recife (PE): contribuição para o programa de controle. J. bras. pneumol. 2006 nov/dez; 32(6): 535-43.

12- Bierrenbach AL, Gomes ABF, Noronha EF, Souza MFM. Incidência de tuberculose e taxa de cura, Brasil, 2000 a 2004. Rev. saúde pública. 2007; 41Supl. 1: 24-33.

13- Ministério da Saúde (BR). Secretaria de Vigilância em Saúde. Departamento de Vigilância Epidemiológica.Manual de Recomendações para o Controle da Tuberculose no Brasil. Brasília (DF): Ministério da Saúde; 2011.

14- Picon PD, Bassanesi SL, Caramori MLA, Ferreira RLT, Jarczewski CA, Vieira PRB. Fatores de risco para recidiva da tuberculose. J. bras. pneumol. 2007set/out; 33(5): 572-8

15- Sanchez AIM, Bertolozzi MR. Além do DOTS (Directly Observed Therapy) no controle da tuberculose: interface e compartilhamento de necessidades. Rev. Latino-Am. Enfermagem. 2009 set/out; 17(5): 68994.

16-Bertolozzi MR. A adesão ao tratamento da Tuberculose na perspectiva da estratégia do Tratamento Diretamente Observado ("DOTS") no município de São Paulo [livre-docência]. São Paulo (SP): Escola de Enfermagem/ USP; 2005
17- Figueiredo TMMR, et al. Desempenho da atenção básica no controle da tuberculose. Rev. saúde pública. 2009 set/out; 43(5): 825-31.

18- Scatena LM, Villa TCS, Ruffino-Netto A, Kritiski AL, Figueiredo TMRM, Vendramini SHF, et al. Dificuldades de acesso a serviços de saúde para diagnóstico de tuberculose em municípios do Brasil. Rev. saúde pública. 2009 maio/jun; 43(3): 389-97.

19- Muniz JN, Palha PF, Monroe AA, Gonzales RIC, Ruffino-Netto A, Villa TCS. A incorporação da busca ativa de sintomáticos respiratórios para 0 controle da tuberculose na prática do agente comunitário de saúde. Ciênc. saúde coletiva. 2005 abr/jun; 10(2): 315-21.

20- Cantalice Filho JP. Efeito do incentivo alimentício sobre o desfecho do tratamento de pacientes com tuberculose em uma unidade primária de saúde no município de Duque de Caxias. J. bras. pneumol. 2009 out; 35(10): 992-7. 